\title{
Cosmovisiones Maipure-Arawakas del Noroeste Amazónico: relaciones de oralidades y escritura desde la interculturalidad
}

\author{
Visões do mundo Maipure-Arawakas do Noroeste Amazônico: relações das \\ oralidades e escrita na interculturalidade \\ Cosmovisions Maipure-Arawakas of the Northwest Amazon: relationships of \\ oralities and writing from the interculturality
}

Omar González Náñez

\section{Resumen}

La cosmovisión que comparten los pueblos etnográfica y lingüísticamente clasificados como "Maipure-Arawakos" (Venezuela) nos permitirá profundizar este conocimiento el cual hemos podido investigar y practicar a través de discursos orales manejados por chamanes y dueños de conocimientos y sus narrativas sobre el significado simbólico de los códigos representados en su arte rupestre, especialmente en sus petroglifos sagrados.

Palabras clave: Arte rupestre. Ceremonias. Cosmovisión.

\section{Características básicas del chamanismo en la cosmovisión de los arawakos del noroeste amazónico}

Con el objeto de hablar de las cosmovisiones que nos han enseñado los sabios indígenas, nos referiremos especialmente al caso de los chamanes Arawakos del Guainía-Río Negro que ocupan territorios ancestrales dentro del noroeste amazónico, por el Rio Negro, entre el sureste del estado amazonas de Venezuela, y la frontera del Sur Colom-

Doctor en Antropología, por la Universidad de Los
Andes, Edo. Mérida, Venezuela, donde es investiga-
dor y docente. Ha sido Profesor invitado de postgra-
do en Antropología y Etnolingüística, Universidad
Central de Venezuela. Investigador de campo en et-
nografía y lingüística de Pueblos Maipure-Arawakos
de la Orinoquia. Miembro del Grupo de Trabajo e
Pueblos Indígenas (CEPSAL-ULA). Indigenista.
E-mail: wamudana@gmail.com

Recebido em 12.08.2018 - Aprovado em 23.09.2018

http://dx.doi.org/10.5335/hdtv.19n.1.8930 
bia por el Guainía y el noroeste del estado Amazonas de Brasil.

El legítimo chamán arawako, en cualquiera de los casos de las etnias que mencionamos acá, es un representante en el mundo de las gentes del Creador del Cosmos para los Arawakos del S. O. Amazónico: Kúwai/ Iñápirrikuli, para los Curripacos; y Nápirùli, Kúwe, Kúwai, Kuwaiseiri, para los Warekena, Baniva, Baré, Piapoco, Yavitero. El protagoniza las sagas más relevantes que su héroe cultural llevó adelante durante los comienzos del Mundo.

Para estas sociedades, “El Ombligo del Mundo", es decir, el lugar donde comenzó la etnogénesis se localiza en un raudal del rio Aiarí, conocido con el nombre de Jípana, en las riberas del caserío de Wapúi. Por esa razón a ese sector también se le conoce como "Wapuí cachoeira" (raudal de Wapuí). El Aiarí es un afluente del curso superior del río Isana, Alto Rio Negro en el Estado de Amazonas, Brasil.

Esta región, como ya lo hemos señalado, forma parte de lo que los etnógrafos han llamado "El área cultural del Noroeste Amazónico" (GOLDMAN, 1968) Bajando de Wapúi se encuentra inmediatamente un segundo raudal llamado Kupí cachoeira. Toda el área (casi unos dos kms.), está cubierta de lajas en las cuales los arawakos del origen dejaron representados por medio de petroglifos y geoglifos el Panteón primordial de los Arawakos. Según el ex-capitán o tuxáua de Wapuí el curripaco Manuel Da Silva (Mandú), (de dialecto õjo-kárru), quen es (aun vive) el último chamán-Tigre de los Curripaco), el Primer Mundo lo conformaban los alrededores del raudal de Jípana pero no había río y se podía abarcar con la vista, es decir, era muy pequeño. En Jípana se localizan una serie de depresiones parecidas a unos pequeños cráteres, ollas o buracos, por esos buracos fue que El Creador tiró de los cabellos a las primeras gentes, los seres humanos que vivían en un inframundo subterráneo. Como ocurre con muchas divinidades de la creación en la historia de la humanidad, Iñápirrikuli es el principio y el fin de todo. A él no se le conocen progenitores. El encerraba las fuerzas vitales de la creación y de la destrucción. En la brumosa génesis del tiempo arawako, él encarnaba a la vez a los dos sexos (era Iñápirrikuli simultáneamente Macho y Hembra). El fue capaz de auto-procrearse. Las versiones de los viejos o "antigüeros" y sabios arawakos mencionan que durante la época de los Dioses, Iñápirrikuli se procuro a una mujer llamada Ámarru. Según algunas versiones ella era una "tia" del Creador. En otras se le menciona como "su mujer". El caso fue que Iñápirrikuli quiso tener un hijo de Ámarru y la preñó sólo con su pensamiento. Ámarru quedó encinta de Kúwai pero como no tenía sexo, debió utilizar para esvirgarla espinas de patáwa (en otras versiones se afirma que la vagina de la mujer fue abierta por un pez mataguaro siguiendo las órdenes del Creador, y por esa razón es que ese pez es de color rojizo ya que fue él “...el que esvirgó a Ámarru".

Antes que Iñápirrikuli creara a los humanos propiamente dichos, en su tiempo convivían espíritus, animales pensantes y gentes. Los humanos solo fueron creados a imagen y semejanza de Kúwai, el Hijo del Creador, una vez que Iñápirrikuli lo des- 
truyó con una gran hoguera ya que el fuego era el único elemento que lo destruía, el espíritu de Kúwai se marchó hacia el quinto y último cielo, y en el lugar donde fue quemado, con sus cenizas Iñápirrikuli formó a los humanos. Los linajes humanos fueron extraídos de los huecos localizados en Jípana. Allí también en Jípana fue donde quemaron a Kúwai, y en el sitio donde lo quemaron nacieron dos plantas absolutamente sagradas con las cuales los hombres actuales elaboran los botutos o flautas de Kúwai (llamadas "diablos" por misioneros y conversos). Las flautas son el cuerpo de Kúwai y todo ritual de iniciación debe estar precedido de las melodías sagradas (o "idioma del Kúwai") que emanan de interior de dichos instrumentos sagrados.

Una versión mítica sobre la etnogenesis arawaka nos habla del orígen de los Hohódeni, el primer linaje arawako (del sub-grupo dialectal õjo-kárru) a ser creado por lo cual lo hemos considerado como una protolengua (GONZÁLEZ ÑÁÑEZ, 1984). No obstante, en esta versión los hohodeni aparecen en el medio del orden de los linajes, ya que antes de ellos se menciona a los Mauliéni (nietos de la avispa). Debemos precisar que en nuestras grabaciones siempre aparecen mencionados de primero los Hohódeni. Mito M.3.0. (WRIGHT, 1981, p. 558-559).

Iñápirrikuli saca a las Gentes: Los Ancestros de los Hohódeni "Iñápirrikuli y el Sol Primigenio (Haáre) buscaba a la gente en Hipana, en el actual rio Aiari". Ellos los sacaron de los huecos del lugar que conduce directamente al "Mundo de abajo" (Wapína-kua). Antes de empezar a buscar, Iñá- pirrikuli quemó toda la tierra con miras a deshacerse de todas las enfermedades y de los seres que las originaban (espíritus de la selva, animales, diablos, etc.) y que estaban en el mundo entonces. Entonces buscaron por los ancestros de las gentes. Se pararon en los huecos del raudal, y en la medida en que Iñápirrikuli daba vuelta a su dedo en círculos, alrededor de cada buraco, surgía un ancestro. El ancestro de cada linaje aparecía con una flauta sagrada de Kúwai en su cabeza, cantando el nombre de su sib mientras iba saliendo del hueco.

Surgieron cinco linajes, todos considerados como hermanos de los Hohódeni. Los hohódeni "aparecieron en el medio del orden y vieron este mundo cuando era el sol del mediodía". Según la versión Hodódeni que maneja R. Wright (1981, p. 558), este subgrupo dialectal curripaco surgiría junto con otros cuatro linajes, conformando una fratria de cinco silabas.

Luego, también en Jípana pero en diferentes buracos irían apareciendo el resto de los linajes con los cuales si se podían casar los Hodódeni (exogamia de clan); por ejemplo, aparecieron los Wariperi-dakénai (nietos de la constelación de las pléyades), también hablantes de curripaco. En ese mismo orden fueron surgiendo, no solo el resto de los curripacos, sino todas las demás lenguas arawakas e incluso las de otras gentes no arawakas como los Tukano, Caribe, Tupi, etc., hasta nombrar todas las gentes del Mundo, incluyendo a los "blancos" o no indígenas.

Las investigaciones llevadas a cabo por investigadores como Silvia Vidal $(1993,2000)$ y Omar González Náñez (1968, 1980, 1984, 
1986, 2007) durante varios años entre grupos Maipure del Norte que habitan la región Isana-Vaupés, han permitido establecer algunos hechos importantes:

1. Los descendientes de diversos de estos grupos, por ejemplo, Curripaco, Warekena, Piapoco, Baniwa y Baré, indican que algunas de las manifestaciones de arte rupestre de esta zona se relacionan con sus antepasados.

2. La localización geográfica y la simbología de estas manifestaciones, hitos geográficos $\mathrm{y} / \mathrm{o}$ marcas asociadas, se vincula con: a) la historia (mítica y real) de estos grupos, b) sus territorios ancestrales y/o tradicionales, c) sus ceremonias de iniciación, d) los nombres de sus fratrias y sibs, e) sus migraciones ancestrales, y f) sus procesos de recreación societaria.

3. En el pasado su simbología y secuencia de lectura era de dominio generalizado, pero con el tiempo este conocimiento se ha ido restringiendo y se limita en la actualidad a los especialistas mágico-religiosos y a los iniciados de sexo masculino.

4. Debido a su importancia para la gente Maipure del Norte, los lugares en donde se encuentran estas manifestaciones son visitados regularmente con el objeto de recordar, reforzar y enseñar la historia (mítica y real), los principios y normas que rigen el funcionamiento de la sociedad y posibilitan su reproducción y continuidad.

Se ha señalado que en las sociedades que carecen de escritura, otros tipos de representaciones gráficas adquieren impor- tancia para transmitir ciertas ideas. Cuando ello ocurre, estas representaciones son semióticas, porque se convierten en sistemas de signos que están estructurados de acuerdo a ciertas reglas de coherencia interna que aseguran descendientes actuales de ciertos grupos Maipure atribuyen a determinados conjuntos de hitos geográficos, marcas y petroglifos de la región Isana-Vaupés y la región Orinoco-Casiquiare-Río Negro. Esta relación solo la pueden hacer los sabios y chamanes en sus narrativas orales, codificadas en sus petroglifos que son sus mecanismos escriturales originarios. Las mujeres, por razones culturales, son excluídas del conocimiento y las prácticas chamánicas y esto se aplica a prohibiciones que aplican a otros pueblos con los cuales están articulados interculturalmente.

Por otra parte, en su contexto de relaciones interculturales con el mundo criollo y sobre todo, en las escuelas misionales o no, han aprendido a escribir el castellano o el portugués (en el caso de Brasil); asimismo, a través de las escuelas interculturales bilingües del Ministerio de Educación han aprendido y usan sistemas escriturales en sus lenguas indígenas, la mayoría diseñados por lingüistas occidentales. No obstante, ni en los alfabetos traídos por la educación occidental (Régimen del EIB) o escritura castellana estándar o en sus lenguas originarias está permitido transcribir y divulgar muchos pasajes de sus mitos por ser muy sagrados. Lamentablemente en la actualidad han desaparecido muchos de esos sabios y las jóvenes generaciones, cada vez más occidentalizadas, ya no participan de la transmisión de los saberes que adquirían a través 
de sus payés, malirris o chamanes. Ya no se practican los rituales de iniciación que todavía documenté y sobre los que escribí en el año 1970 cuando viví en Wayanápi entre los warekena.

Hay otra reflexión en torno a la oralidad y que destaca el papel que desempeña esa práctica en pueblos de tradición no escrita en la lengua dominante colonial y es el hecho que destacan muchos autores y escritores indígenas o no (como A. Roa Bastos, F. Fanon, H. Camico. entre otros) sobre el hecho de nombrar o significar mediante segundas lenguas impuestas o coloniales y el uso preferencial, casi automático, de sus lenguas maternas. Utilizaremos un ejemplo tomado de Mosonyi (2005) refiriéndose a un hablante bilingüe baniva-castellano, el Sr. Hernán Camico.

Nuestro colaborador fallecido, el señor Hernán Camico, era perfectamente bilingüe en baniva y castellano, e incluso utilizaba con más frecuencia y en mayor número de situaciones el idioma oficial que el originario. Sin embargo, cuando le solicitamos nombrar todas las especies de vegetales de las que tuviese conocimiento, primero las enumeraba en ambas lenguas, pero pronto llegó el momento en que tuvo que continuar forzosamente en baniva. Él podía nombrar y hasta describir centenares de especies biológicas pero sólo en su lengua materna y en los parámetros de su cultura indígena. Lo propio sucedía con los nombres, la elaboración y los usos de los diferentes objetos que constituyen la cultura material tradicional de este pueblo. Él podía nombrar y caracterizar, dentro de su proceder etnocientifico, todos estos enseres: algo que jamás podría haber hecho en castellano, a pesar de su dominio pleno de este idioma. Cuando pasamos de la cultura tangible a la intangible, la dificultad de operar con el castellano se hacía mucho mayor todavía, ya hasta los límites de lo absurdo. Así que ni siquiera el bilingüe perfecto puede disociar su cultura nativa del idioma que legítimamente le pertenece.

Invito a nuestros lectores a apoyarnos en plantear o sugerir posibles soluciones educativas para frenar este etnocidio.

\section{Resumo}

A visão de mundo compartilhada etnografica e linguisticamente classificada como "Maipure-Arawakos" (Venezuela) nos permite aprofundar o conhecimento, que pudemos investigar e praticar por meio de discursos orais administrados por xamãs e donos de conhecimento e de suas narrativas sobre o significado simbólico dos códigos representados em sua arte rupestre, especialmente em seus petróglifos sagrados.

Palavras-chave: Arte rupestre. Cerimônias. Visão de mundo.

\section{Abstract}

The worldview shared ethnographically and linguistically classified as "Maipure-Arawakos" (Venezuela), will allow us to deepen this knowledge, which we have been able to investigate and practice through oral discourses managed by shamans and knowledge owners and their narratives about the symbolic mea- 
ning of the codes represented in their rock art, especially in their sacred petroglyphs.

Keywords: Rock art. Ceremonies. Worldview.

\section{Referencias}

GOLDMAN, Irving. Los Cubeo. Indios del Noroeste del Amazonas. México: Ediciones del Instituto Indigenista Interamericano. 1968

GONZÁLEZ ÑÁÑEZ, Omar. La mitología baniva reflejada en su literatura oral. Revista Economia y Ciencias Sociales. Editorial Faces-UCV, Caracas, a. X, n. 3, p. 87-96, 1968.

Ávila, 1980.

Mitologia warekena. Caracas: Monte . Algunos problemas de reconstrucción y correspondencia de sonidos en la familia lingüística Arawaka. Trabajo de Ascenso. Caracas: Ediciones FACES-UCV, 1984.

Sexualidad y Rituales de Iniciación entre los indígenas Warekena del Río Guainía-Río Negro. Separata de la revista Montalban, Universidad Católica Andrés Bello, Caracas, n. 17, p. 103-138, 1986.

Las literaturas indígenas maipure-arawakas de los pueblos kurripako, warekena y baniva del estado amazonas. Caracas: Fundación Editorial El Perro y la Rana, 2007.

MOSONYI, Esteban. En defensa de la multiculturalidad: Nuestra diversidad lingüística indígena y vernácula. Revista Question, Caracas, v. 10, n. 2, p. 28-55, 2005.

VIDAL, Silvia M. Reconstrucción de los procesos de etnogenesis y de reproducción social entre los baré de río negro (siglos xvi-xviii). Tesis Doctoral. Caracas: Ediciones IVIC, 1993.
. Historia y Etnicidad en el Noroeste Amazónico. Mérida: Consejo de Publicaciones de la Universidad de Los Andes, 2000.

WRIGHT, Robin. History and Religion of the Baniwa people of Upper Rio Negro Valley. PhD Dissertation thesis, Stanford University, Stanford, USA, 1981. 